\title{
Psychiatrische Klassifikationssysteme und Forensische Psychiatrie
}

\author{
Henning Saß
}

Online publiziert: 22. juli 2015

(C) Springer-Verlag Berlin Heidelberg 2015

Die Diagnose stellt eine entscheidende Grundlage allen ärztlichen Denkens und Handelns dar. In der Psychiatrie allerdings galt die Diagnostik zu Recht lange als Schwachstelle, weil sie in hohem Maße schulengebunden, wenig reliabel und international nicht vergleichbar war. Eine fundamentale Änderung dieser klinisch wie wissenschaftlich unbefriedigenden Situation setzte jedoch mit der Einführung der operationalisierten Klassifikationssysteme ein. Das von der Amerikanischen Psychiatrischen Vereinigung herausgegebene DSM-III ${ }^{1}$ (1980, deutsch 1984) enthielt erstmals für das Gesamtgebiet der psychischen Störungen explizite diagnostische Kriterien. Diese bezogen sich auf Art, Anzahl und Dauer der Symptome oder auf Schweregrad oder Verlaufsform des Symptomenbildes und waren in exakten Algorithmen systematisch verknüpft. Des Weiteren wurde ein multiaxiales Diagnosesystem eingeführt, das den Patienten auf 5 Achsen - psychiatrische Diagnose, Persönlichkeit, sonstige medizinische Erkrankungen, lebenssituative Umstände und allgemeines Funktionsniveau - beurteilte.

Die Beschreibungen der Störungen in DSM-III sollten weitgehend unabhängig von ätiologischen Theorien sein, sodass z. B. auch nicht mehr zwischen neurotischen bzw. endogenen Störungen unterschieden wurde. Um Objektivierbarkeit zu gewährleisten, sollten für die Kriterienlisten vorwiegend solche Symptome ausgewählt werden, die möglichst von außen beobachtbar waren. In DSM-III-R (R: „revised“, 1987, deutsch 1989) wurde zudem das Komorbiditätsprinzip eingeführt, d. h., bis dahin gültige diagnostische Hierarchieregeln wurden weitgehend aufgehoben, sodass beim selben Patienten mehrere Diagnosen nebenei-

${ }^{1}$ Diagnostic and Statistical Manual of Mental Disorders, 3. Ausgabe.

H. Saß $(\bowtie)$

Aachen, Deutschland

E-Mail: hsass@ukaachen.de nander vergeben werden können, wenn die entsprechenden Kriterien erfüllt sind. Das wesentlich umgestaltete DSM-IV (1994, deutsch 1996) integrierte die umfangreichen Erträge der Diagnostikforschung seit Erscheinen von DSM-III. DSM-IV-TR (TR: „text revision“, 2000, deutsch 2003) enthielt dann bei unveränderten diagnostischen Kriterien eine Aktualisierung der beschreibenden Texte aufgrund neuerer empirischer Daten sowie eine weitere Vermehrung der Störungskategorien durch zusätzliche Unterformen.

Das neue DSM-5, erschienen in den USA im Mai 2013 und in Deutschland im Dezember 2014 [1, 2], geht auf einen 12-jährigen, intensiven Revisionsprozess zurück und enthält zahlreiche wichtige, z. T. grundlegende Änderungen [4]. So sind die Hauptgruppen von Störungen in Orientierung am Lebenszyklus angeordnet, von den Erkrankungen im Kindesund Jugendalter zu den meist im späten Erwachsenenalter auftretenden neurokognitiven Störungen. Integriert wurden aktuelle Entwicklungen und Befunde der Neurowissenschaften. Neu formiert wurde eine Gruppe der „Autismus-Spektrum-Störungen“. Die Klassifikation der bipolaren und der depressiven Störungen wurde anders gegliedert, ebenso die der Störungen im Zusammenhang mit psychotropen Substanzen. Der Demenzbegriff wurde aufgegeben zugunsten eines neuen Begriffs der neurokognitiven Störungen. Ein „Alternatives Modell für Persönlichkeitsstörungen“ wurde vorgeschlagen und ein Abschnitt für neue Erscheinungsformen mit weiterem Forschungsbedarf angefügt.

Was bedeuten nun diese im Hauptfach viel beachteten und folgenreichen Entwicklungen für die Subspezialität der Forensischen Psychiatrie? Natürlich kommt auch bei der psychiatrischen und der psychologischen Begutachtung von Straftaten wie im Zivilrecht der Diagnosestellung eine zentrale Bedeutung zu. Entsprechend wird in den Leitlinien und Mindeststandards unseres Faches die Orientierung an den aktuellen Systemen von ICD und DSM empfohlen. 
Allerdings muss gerade im forensischen Kontext vor einer Überschätzung der diagnostischen Begriffe und Kodierungen gewarnt werden. In Laienhand und bei unreflektierter Anwendung droht die Gefahr einer Reifizierung der diagnostischen Termini. Demgegenüber ist für die allermeisten psychischen Störungen zu betonen: Die dafür geläufigen Diagnosen stellen keine in naturwissenschaftlichem Sinne objektivierbaren Tatsachen dar; vielmehr handelt es sich um zeitgebundene Konventionen zur Verständigung über abnorme psychische Phänomene.

Es unterliegen nämlich, wie es am Beispiel des DSMSystems skizziert wurde, die Auffassungen über klassifikatorische Prinzipien, nosologische Gruppierungen, diagnostische Algorithmen und symptomatologische Kriterien einem ständigen, zumeist wissenschaftsgetriebenen Wandel. Juristischen Beurteilern macht dies z. B. dann Schwierigkeiten, wenn bei längeren Verläufen im Maßregelvollzug die diagnostischen Bezeichnungen der beteiligten Therapeuten und Gutachter variieren. Oft beruht dies eher auf semantischen Gründen und nicht auf einer wesentlichen Änderung zugrunde liegender psychopathologischer Sachverhalte. Bei der Schuldfähigkeitsprüfung ergibt sich darüber hinaus das Problem, dass die rechtlichen Vorgaben auf möglichst trennscharfe kategoriale Unterscheidungen ausgerichtet sind. Dies ist nur schwer mit einer dimensionalen Konzeptualisierung von psychischen Funktionen und ihren Störungen zu vereinbaren, die v. a. unter dem Einfluss neurowissenschaftlicher Forschungsstrategien zunehmend zum Rationale diagnostischer Systeme gemacht wird, neuerdings anspruchsvoll als Präzisionsmedizin für die Psychiatrie formuliert [3].

Verstärkt werden interdisziplinäre Friktionen durch Überdehnungen der Krankheits- und Störungsbegriffe teils aus dem eigenen Fach heraus, teils unter dem Einfluss rechtspolitischer Interessen, wie es zuletzt am Beispiel des diagnostisch nahezu nichtssagend gewordenen Terminus der „psychischen Störung“ geschehen ist (zur Kritik: [5, 7, 8). Umso wichtiger erscheint es aus forensischer Perspektive, bei unserem diagnostischen Vorgehen am Konzept einer Differenzierung zwischen psychopathologisch bedeutsamen Auffälligkeiten einerseits und dissozialer bzw. krimineller Devianz andererseits festzuhalten, dies auch im Interesse des Schutzes psychisch Kranker. Anderenfalls kommt es zu dem Fehlschluss, immer weitere Bereiche sozial abweichenden, störenden oder gefährlichen Verhaltens zum Beleg für psychische Störung zu nehmen.

Inflationäre Tendenzen in der aktuellen Diagnostik psychischer Störungen beruhen darüber hinaus auf dem Komorbiditätsprinzip und der ständig wachsenden Zahl diagnostizierbarer Störungsbilder in den aufeinanderfolgenden Auflagen der Klassifikationssysteme. Dies beruht oft weniger auf Wissensfortschritt als auf speziellen Forscherinteressen oder auf ökonomischen Zielen. Wegen der bedeutsamen Unterschiede zwischen den klassifikatorischen Gebräuchen in der allgemeinen Psychiatrie und den speziellen forensisch-psychiatrischen Problemstellungen enthält DSM-5 deshalb, wie alle früheren Auflagen, einen dezidierten Warnhinweis für die Anwendung in rechtlichen Zusammenhängen. Denn auch, wenn gemäß ICD oder DSM die Diagnose einer psychischen Störung zu vergeben ist bzw. deren mehrere kodiert werden können, was in letzter Zeit immer häufiger möglich wird, folgen erst dann die entscheidenden Schritte: das Herausarbeiten eines Zusammenhanges zwischen der Entstehung des Delikts und den Symptomen der psychischen Störung sowie die Diskussion von deren Schwere und Auswirkungen zur Tatzeit. Für die gutachterliche Einschätzung ist daher wichtiger noch als die Diagnosestellung die forensisch-psychopathologische Analyse von diagnostizierter Störung und Delikt [6].

In neuerer Zeit allerdings treten zu der vorwiegend psychopathologisch orientierten Diagnostik psychischer Störungen immens anwachsende Kenntnisse etwa über die neurobiologischen und psychophysiologischen Grundlagen von Aggressivität, Impulsivität und Verhaltenskontrolle hinzu. Sie erweitern unser Verständnis von delinquenten Verhaltensweisen insbesondere bei Patienten mit Persönlichkeitsstörungen und sexuellen Deviationen sowohl in ätiopathogenetischer wie in prognostischer und therapeutischer Hinsicht, werfen aber auch neue Fragen zur Problematik des freien Willens und der Verantwortlichkeit auf. Zwar ist bis heute noch keine übergreifende Theorie erkennbar, dennoch werden die zahlreichen strukturellen und funktionellen Befunde der Neurowissenschaften möglicherweise auch die Diagnostik und forensische Bewertung im Sinne einer Ergänzung durch biologische Parameter verändern.

Im Sinne der skizzierten Fragen geht es in den Beiträgen zum Schwerpunktthema dieses Heftes der Zeitschrift Forensische Psychiatrie, Psychologie, Kriminologie um die Bedeutung der Weiterentwicklung der psychiatrischen Diagnostik für die forensische Psychiatrie. So nehmen Müller et al. aus Göttingen Bezug auf die Einführung von psychophysiologischen Verfahren als diagnostische Marker bei der Pädophilie in DSM-5 und diskutieren forensische Konsequenzen aus der empirischen Befundlage hinsichtlich Diagnostik, Verhaltenskontrolle und Prognostik. Briken aus Hamburg beschäftigt sich mit konzeptionellen Aspekten der Kriterien für Paraphilie und paraphile Störungen in DSM-5 und weist aus sexualwissenschaftlicher Perspektive auf Risiken der neuen Definitionen hin. Auch Kröber aus Berlin sieht bei der Diskussion des schwierigen Kapitels über Disruptive, Impulskontroll- und Sozialverhaltensstörungen erhebliche Probleme in der Konzeption wie in der Aussagekraft solcher empirisch-deskriptiv lediglich auf Verhaltensweisen basierten Diagnosen für die forensischpsychiatrischen Fragestellungen. 
Als weiterer ,hot spot“ forensischer Aufgabenstellungen folgt die Einführung von Berberich und Zaudig aus Windach in das Alternative Modell für Persönlichkeitsstörungen des DSM-5, das zwar initial enorm elaboriert und kompliziert anmutet, aber doch wichtige empirische Neuerungen und dringend benötigte Möglichkeiten standardisierter Erfassung enthält. Tribolet-Hardy et al. aus der Züricher Arbeitsgruppe diskutieren vor dem Schweizer Hintergrund Tendenzen zur Ausweitung des Krankheitsbegriffes und Pathologisierung delinquenten Verhaltens, mit der möglichen Konsequenz weitgehenden Verzichtes auf diagnostische Fundierungen forensischer Akte. Die lange Geschichte der Auffassungsunterschiede zwischen Juristen und Medizinern über Krankheitsbegriffe und die Nöte beim Umgang mit rechtlichen Schwellenvorgaben stellt Nedopil aus München dar.

Sodann folgt im Nachgang zum vorigen Heft von Forensische Psychiatrie, Psychologie, Kriminologie eine Arbeit zum Themenkreis von Verantwortung, Schuld und Wahrheit. Vor dem Hintergrund seiner seelsorgerischen Arbeit spannt Mundhenk aus religiöser Perspektive das Thema von Schuld und Vergebung in forensischem Kontext aus, das bis hin zur problematischen Position Psychiatrieerfahrener reicht, die für psychisch kranke Menschen im Hinblick auf die Schuldfähigkeit völlige Gleichbehandlung und mithin ein Absehen von krankheits- und diagnosebezogenen Rücksichten proklamieren.

Wie wird es in der psychiatrischen Diagnostik weitergehen? Klinisch und wissenschaftlich hinderlich erscheint, auch in der Forensik, die Doppelung in einerseits die ICD-10 bzw. in etwa 3 bis 4 Jahren dann die ICD-11 und andererseits das DSM-5 mit späteren Revisionen. Zwar ist zu erwarten, dass beide Systeme in enger Abstimmung weiterentwickelt werden, doch bleibt es aus verschiedenen Gründen zweifelhaft, dass sie jemals vollständig in Übereinstimmung gebracht werden. Zum einen hat die Weltgesundheitsorganisation (WHO) mehr Gewicht auf klinische Brauchbarkeit und internationale Anwendbarkeit zu legen, während es sich bei DSM-III/IV/5 um ein nationales System mit allerdings internationalen und hegemonialen Ansprüchen handelt. Auch wird die ICD, die rationell und alltagstauglich abgefasst sein muss, um dem Anwender in Klinik und Praxis einen raschen Überblick bei der Dokumentation zu geben, nie den Umfang an Informationen bieten können, die den verschiedenen Auflagen des stark auf Forschung gestützten DSM einen fast lehrbuchartigen Charakter gibt. Darüber hinaus hat die WHO bei der Abfassung des Kapitels V (F) Rücksicht auf die Struktur und Verschlüsselungsvorschriften anderer medizinischer Disziplinen zu nehmen, um die Einheitlichkeit der ICD zu gewährleisten. Schließlich sind auch finanzielle Gründe nicht zu unterschätzen, dürfte das DSM-IV mit den entsprechenden Beigaben (Handbuch der Differentialdiagnosen, Kriterienhandbuch, Quellenverzeichnis, Fallbuch, Klinische Interviews etc.) doch eines der auflagen- und umsatzstärksten medizinischen Werke weltweit gewesen sein, was auch für DSM-5 erwartet werden kann.

Die rasche weltweite Akzeptanz der operationalisierten Klassifikationssysteme in den letzten 3 Jahrzehnten zeigt im Nachhinein, wie groß der Bedarf für eine Vereinheitlichung der psychiatrischen Diagnosesprache war. Allerdings bleibt es wohl auch künftig bei einer Diskrepanz zwischen einerseits der Komplexität des psychischen Geschehens im lebenssituativen Kontext des Patienten und andererseits der Tendenz zu Vereinfachung und Reduktion des psychiatrischen Denkens und Diagnostizierens. Diese ist den operationalisierten Klassifikationssystemen grundsätzlich inhärent, doch begrenzt gerade die Reduktion auch den Nutzen für die Forensische Psychiatrie.

Interessenkonflikt $\mathrm{H}$. Saß gibt an, dass kein Interessenkonflikt besteht.

\section{Literatur}

1. APA (American Psychiatric Association) (2013) Diagnostic and statistical manual of mental disorders (DSM-5). American Psychiatric Publishing, Arlington

2. Falkai P, Wittchen H-U, Doepfner M, Gaebel W, Maier W, Rief W, $\mathrm{Sa}$ H, Zaudig M (Hrsg) (2015) Diagnostisches und Statistisches Manual Psychischer Störungen DSM-5. Hogrefe, Göttingen

3. Insel TR (2014) The NIMH Research Domain Criteria (RDoC) project: precision medicine for psychiatry. Am J Psychiatry 171:395-397

4. Jäger M (2015) Aktuelle psychiatrische Diagnostik. Ein Leitfaden für das tägliche Arbeiten mit ICD und DSM. Thieme, Stuttgart

5. Kröber H-L (2011) „Psychische Störung“ als Begründung für staatliche Eingriffe in Grundrechte des Individuums. Forens Psychiatr Psychol Kriminol 5:234-243

6. Saß H (2008) Psychische Störungen und Schuldfähigkeit - Ein psychopathologisches Referenzsystem. Psychiatrie 5(3):182-189

7. Saß H (2011) Psychische Störung zwischen Medizin und Recht: Anything goes? Forens Psychiatr Psychol Kriminol 5:129-131

8. $\mathrm{Sa} ß \mathrm{H}$ (2015) Persönlichkeit und Verantwortung. Forens Psychiatr Psychol Kriminol 9:75-81 